\title{
Green's-function solution for a special class of master equations
}

\author{
Otto J. Eder and Thomas Lackner* \\ Austrian Research Center Seibersdorf, A-2444 Seibersdorf, Austria \\ Maximilian Posch \\ International Institute for Applied Systems Analysis, A-2361 Laxenburg, Austria
}

(Received 1 August 1984)

\begin{abstract}
We consider a one-dimensional stochastic process described by a master equation and calculate the time-dependent distribution function. The time evolution of the system is given by the conditional probability $h\left(x, t \mid x_{0}\right)$, where $x$ and $x_{0}$ are continuous variables. Assuming a quite general dependence of the transition probability $W_{\Omega}\left(x \rightarrow x^{\prime}\right)$ on a parameter $\Omega$, we show that the backward form of the master equation can be used to calculate arbitrary conditional averages $\left\langle f(x) \mid x_{0}\right\rangle_{t}$ up to any given power in $\Omega^{-1}$. This general expansion procedure will be used to construct $h\left(x, t \mid x_{0}\right)$ itself. We show that-introducing a new stochastic variable $y$-the conditional probability $h\left(x, t \mid x_{0}\right)$ can be expanded into a series of Hermite functions. The coefficients of this expansion, $b_{n}(t)$, which depend on the expansion parameter $\Omega$, can be uniquely determined via a recursion relation. We show that in the limit $\Omega \rightarrow \infty$ all coefficients $b_{n}(t)$ vanish, except $b_{0}$, which is time independent. In this limit a Gaussian distribution for the conditional probability is obtained, which is in agreement with the so-called linear noise approximation.
\end{abstract}

\section{INTRODUCTION}

A common formulation of stochastic processes is given in terms of a hierarchy of various probabilities, i.e., singlet, joint, and conditional probabilities. ${ }^{1}$ One can either derive exact equations for the singlet probability involving also the conditional probabilities, or one can derive an open hierarchy for the conditional probabilities alone. Although these equations look formally very simple, they cannot be solved in general, since the equation for the lower-order conditional probability requires the knowledge of a higher-order probability. In order to avoid these difficulties one assumes that the higher-order conditional probabilities are identical to the lowest-order probability, which we will call $h\left(x, t \mid x_{0}\right)$. A process based on these assumptions is called a Markov process ${ }^{1,2}$ and the time evolution of the conditional probability $h\left(x, t \mid x_{0}\right)$ can then be described by an integro-differential equation, which is commonly called Markov or master equation. The central quantity entering the master equation is the transition probability $W_{\Omega}\left(x \rightarrow x^{\prime}\right)$, which in general also depends on time. However, in many physical applications the transition probability can be considered as time independent, leading to a Markov process homogeneous in time. It is exactly this process, which will be considered in this paper for the case of a one-dimensional, continuous, unbounded stochastic variable $x$.

A variety of physical problems can be formulated in terms of a master equation. They are found in transport problems, the study of gas-phase relaxation, ${ }^{3}$ reaction $\mathrm{ki}$ netics, ${ }^{4}$ birth and death processes, random walks, ${ }^{5}$ etc. In each particular application physical intuition and/or first-principles calculations have to be used in order to arrive at an explicit expression for the transition probability $W_{\Omega}\left(x \rightarrow x^{\prime}\right)$, which determines the entire process. It turns out that in only very few examples the transition probability allows an analytic solution of the master equation. Furthermore-except in the case of linear one step processes-no rigorous technique exists for solving master equations. This difficulty is the reason why-instead of looking for an analytic solution-one tries to find approximations to the original master equation. The well-known Kramer-Moyal expansion, ${ }^{6}$ e.g., transforms the master equation into a partial differential equation of infinite order, which involves the jump moments $\alpha_{n}(x)$ of the transition probability. If one then assumes that the moments $\alpha_{n}$ with $n \geq 3$ are small compared to $\alpha_{1}$ and $\alpha_{2}$ and neglects them one arrives at the nonlinear Fokker-Planck equation. However, if one tries to find higher-order approximations including $\alpha_{3}, \alpha_{4}, \ldots$, one would run into a logical inconsistency as pointed out by Pawula. ${ }^{7}$ The first rigorous transformation of the master equation to a Fokker-Planck type equation was presented by van Kampen. ${ }^{8}$ He assumes a quite general dependence of the transition probability on a physical parameter $\Omega$ and, after introducing a new stochastic variable $y$, he was able to show that in the limit $\Omega \rightarrow \infty$ the original master equation can be replaced by a Fokker-Planck equation with timedependent coefficients. Although this limit is exact, no general procedure or approximation scheme is available for the case where $\Omega$ is finite. A third method, which is closely related to van Kampen's method, was given by Kubo et al. ${ }^{9}$ These authors assume an exponential form $\exp \left[\sum_{l} \Omega^{-1} q_{l}\left(\xi, x_{0}, t\right)\right]$ for the Fourier transform of $h\left(x, t \mid x_{0}\right)$ and show that for $\Omega \rightarrow \infty$ a Gaussian distribution is obtained. In this limit the result is identical to that obtained by van Kampen. This method has the disadvantage that higher approximations for the distribution itself are difficult to find, since the inverse transform of $\exp \left[\sum_{l} \Omega^{-l} q_{l}\left(\xi, \dot{x}_{0}, t\right)\right]$ is not known in general. 
In a previous paper ${ }^{10}$ the present authors have shown that the transport equation, which is the average of the backward master equation, can be used to calculate an arbitrary conditional average $\left\langle f(x) \mid x_{0}\right\rangle_{t}$ systematically as a power series in $\Omega^{-1}$. The method was also used to find an approximation to the distribution function $h\left(x, t \mid x_{0}\right)$ itself, which turned out to be identical with the linear noise approximation obtained originally by van Kampen and Kubo et al. However, from a mathematical point of view this approximation is unsatisfactory, since it results from neglecting certain terms in our expansion rather than being a limiting process in a mathematical sense. The purpose of this paper is to go beyond the linear noise approximation and to present the complete solution to the master equation. From this general solution one can then easily find the mathematical limitations under which the Gaussian distribution is obtained. Introducing a new stochastic variable $y$ (see Sec. V) we will show that the distribution function $h\left(x, t \mid x_{0}\right)$ can be expanded into a series of Hermite functions, $\sum_{n=0}^{\infty} b_{n}(t) \Psi_{0}(y) \Psi_{n}(y)$. It will turn out that the coefficients $b_{n}(t)$, which depend on the parameter $\Omega$, are uniquely determined by recursion relations. In this sense our solution is not a formal solution to the problem and successive approximations can be actually computed by single integrations. Replacing the infinite sum of Hermite functions by a finite sum, $\sum_{n=0}^{k} b_{n}(t) \Psi_{0}(y) \Psi_{n}(y)$, we define the $k$ th approximation to $h\left(x, t \mid x_{0}\right)$. Although we are not able to answer questions concerning the positivity of the $k$ th approximation, one can easily show that the $k$ th approximation reproduces the exact moments $\left\langle y^{l}\right\rangle$ for $0 \leq l \leq k$. Furthermore, in the limit $\Omega \rightarrow \infty$, all coefficients $b_{n}(n \geq 1)$ vanish, while $b_{0}$ is a constant. The distribution is then given by the zeroth approximation $\Psi_{0}^{2}(y) / \sqrt{\pi}$, which is identical with the linear noise approximation.

\section{METHOD FOR SOLVING TRANSPORT EQUATIONS}

Although a detailed description of the method for solving transport equations can be found in our previous paper, ${ }^{10}$ we want to repeat the main results for completeness. Let us consider a stationary Markov process for a onedimensional, continuous stochastic variable $x(t)$ with $-\infty \leq x(t) \leq \infty$. The time distribution function (TDF) $h\left(x, t \mid x_{0}\right)$, which is the conditional probability for finding the stochastic variable $x(t)$ between $x$ and $x+d x$ at time $t$, given that at time $t=0$ it had the value $x_{0}$, can be described equivalently ${ }^{10,11}$ by the forward master equation

$$
\begin{aligned}
\frac{\partial}{\partial t} h_{f}\left(x, t \mid x_{0}\right)= & -P_{\Omega}(x) h_{f}\left(x, t \mid x_{0}\right) \\
& +\int W_{\Omega}\left(x_{1} \rightarrow x\right) h_{f}\left(x_{1}, t \mid x_{0}\right) d x_{1}
\end{aligned}
$$

or the backward master equation

$$
\begin{aligned}
\frac{\partial}{\partial t} h_{b}\left(x, t \mid x_{0}\right)= & -P_{\Omega}\left(x_{0}\right) h_{b}\left(x, t \mid x_{0}\right) \\
& +\int W_{\Omega}\left(x_{0} \rightarrow x_{1}\right) h_{b}\left(x, t \mid x_{1}\right) d x_{1}
\end{aligned}
$$

with the initial conditions

$$
h_{f}\left(x, t=0 \mid x_{0}\right)=h_{b}\left(x, t=0 \mid x_{0}\right)=\delta\left(x-x_{0}\right) .
$$

Here $W_{\Omega}\left(x \rightarrow x_{1}\right) d t d x_{1}$ has to be considered as a known function describing the probability for a transition from $x$ to $x_{1}$ in a small time interval $d t$ and $P_{\Omega}(x)$ is given by

$$
P_{\Omega}(x):=\int W_{\Omega}\left(x \rightarrow x_{1}\right) d x_{1} .
$$

The subscript $\Omega$ refers to an explicit dependence of the transition probability on a given parameter $\Omega$. An important quantity, related to the distribution function, is the conditional average of an arbitrary function $f(x)$, defined by

$$
\left\langle f(x) \mid x_{0}\right\rangle_{t} \equiv \chi\left(x_{0}, t\right):=\int f(x) h\left(x, t \mid x_{0}\right) d x .
$$

Comparing Eqs. (1a) and (1b) we see that only the backward form of the master equation provides us with the following integro-differential equation for $\chi\left(x_{0}, t\right)$ (see Refs. 10 and 11):

$$
\frac{\partial}{\partial t} \chi\left(x_{0}, t\right)+P_{\Omega}\left(x_{0}\right) \chi\left(x_{0}, t\right)=\int W_{\Omega}\left(x_{0} \rightarrow x_{1}\right) \chi\left(x_{1}, t\right) d x_{1}
$$

which has to be solved subject to the initial condition

$$
\chi\left(x_{0}, t=0\right)=f\left(x_{0}\right) .
$$

Equation (4a), which is called transport equation, is the starting point for our considerations. Let us assume that the transition probability $W_{\Omega}\left(x_{0} \rightarrow x_{1}\right)$ can be written in the following form: ${ }^{8}$

$$
W_{\Omega}\left(x_{0} \rightarrow x_{1}\right)=F(\Omega) W\left(x_{0}, \Omega\left(x_{1}-x_{0}\right)\right),
$$

where on the right-hand side (rhs) of Eq. (5) the dependence on $\Omega$ appears explicitly and $F(\Omega)$ is a given function. Although the precise definition of the parameter $\Omega$ depends on the system considered, we assume that in our system there exists a size parameter $\Omega$ which scales the jump length. As has been shown previously, ${ }^{10}$ this special dependence of the transition probability on the parameter $\Omega$ can be used for separating the arbitrary conditional average $\chi$ into a nonfluctuating part $\chi_{0}$ and fluctuating parts $\chi_{l}(l \geq 1)$. Expanding $\chi$ in a power series in $\Omega^{-1}$

$$
\chi\left(x_{0}, \tau\right)=\sum_{l=0}^{\infty} \frac{1}{\Omega^{l}} \chi_{l}\left(x_{0}, \tau\right)
$$

we have shown that the nonfluctuating part $\chi_{0}$ is the solution of a homogeneous first-order partial differential equation,

$$
\chi_{0}\left(x_{0}, \tau\right)=f(\bar{x}),
$$

where $\bar{x}$ is the solution of the macroscopic equation

$$
\frac{d \bar{x}}{d \tau}=\alpha_{1}(\bar{x}), \bar{x}(0)=x_{0} .
$$

The fluctuating contributions $\chi_{l}(l \geq 1)$ obey inhomogeneous first-order partial differential equations, the solutions of which are given by

$$
\chi_{l}\left(x_{0}, \tau\right)=\chi_{l}\left[x_{0}, \bar{x}\right]=\int_{x_{0}}^{\bar{x}} \frac{H_{l}[y, \bar{x}]}{\alpha_{1}(y)} d y
$$


with

$$
\begin{aligned}
H_{l}\left(x_{0}, \tau\right) & =H_{l}\left[x_{0}, \bar{x}\right] \\
& =\sum_{s=2}^{l+1} \frac{\alpha_{s}\left(x_{0}\right)}{s !} \frac{d^{s}}{d x_{0}^{s}} \chi_{l+1-s}\left[x_{0}, \bar{x}\right], \quad l \geq 1 .
\end{aligned}
$$

In deriving the above equations we introduced a new time scale

$$
\tau:=\frac{F(\Omega)}{\Omega^{2}} t
$$

and the jump moments of the transition probability

$$
\alpha_{n}\left(x_{0}\right)=\int W\left(x_{0}, y\right) y^{n} d y
$$

which are independent of $\Omega$. Furthermore it turns out that the time dependence of $\chi_{l}\left(x_{0}, \tau\right)$ is given by the solution of the macroscopic equation alone; this is indicated by using the square brackets in Eq. (9a) and (9b).

From Eqs. (7)-(9) we see that the functions $\chi_{l}$ can be determined successively, the calculation of $\chi_{l}$ involving only differentiations of the previous functions $\chi_{k}$ $(k \leq l-1)$ with respect to $x_{0}$. This differentiation can be carried out explicitly, since the dependence of $\bar{x}$ on the initial condition $x_{0}$ is given by ${ }^{10,12}$

$$
\frac{d \bar{x}}{d x_{0}}=\frac{\alpha_{1}(\bar{x})}{\alpha_{1}\left(x_{0}\right)}
$$

which implies for example for the rhs of Eq. (9b)

$$
\frac{d}{d x_{0}} \chi_{l}\left[x_{0}, \bar{x}\right]=\frac{\partial}{\partial x_{0}} \chi_{l}\left[x_{0}, \bar{x}\right]+\frac{\alpha_{1}(\bar{x})}{\alpha_{1}\left(x_{0}\right)} \frac{\partial}{\partial \bar{x}} \chi_{l}\left[x_{0}, \bar{x}\right] .
$$

In our previous paper we have also shown that the dependence of the quantities $\chi_{l}$ on the arbitrary function $f$ can be made explicit, yielding

$\chi_{l}\left[x_{0}, \bar{x}\right]=\sum_{k=0}^{2 l-1} f^{(2 l-k)}(\bar{x}) \chi_{l, 2 l-k}\left[x_{0}, \bar{x}\right], l \geq 1$.

In Eq. (14), $f^{(k)}(\bar{x})$ denotes the $k$ th derivative of $f$ with respect to the argument and $\chi_{l, 2 l-k}$ are functions independent of $f$, containing the moments $\alpha_{n}\left(x_{0}\right)$ only. The importance of Eq. (14) becomes clearer, if we combine it with Eq. (6) to obtain

$$
\begin{aligned}
& \left\langle f(x) \mid x_{0}\right\rangle_{\tau} \equiv \chi\left(x_{0}, \tau\right) \equiv \chi\left[x_{0}, \bar{x}\right]=f(\bar{x})+\frac{1}{\Omega}\left[f^{(2)}(\bar{x}) \chi_{1,2}+f^{(1)}(\bar{x}) \chi_{1,1}\right] \\
& +\frac{1}{\Omega^{2}}\left[f^{(4)}(\bar{x}) \chi_{2,4}+f^{(3)}(\bar{x}) \chi_{2,3}+f^{(2)}(\bar{x}) \chi_{2,2}+f^{(1)}(\bar{x}) \chi_{2,1}\right]+O\left(\frac{1}{\Omega^{3}}\right)
\end{aligned}
$$

or in general

$$
\begin{aligned}
\left\langle f(x) \mid x_{0}\right\rangle_{\tau}= & f(\bar{x}) \\
& +\sum_{l=1}^{\infty} \frac{1}{\Omega^{l}} \sum_{k=0}^{2 l-1} f^{(2 l-k)}(\bar{x}) \chi_{l, 2 l-k}\left(x_{0}, \tau\right) .
\end{aligned}
$$

We see that an arbitrary conditional average can be calculated up to any order in $\Omega^{-1}$, once the functions $\chi_{l, 2 l-k}$ are known. Therefore the functions $\chi_{l, 2 l-k}$ will play an essential role in our further considerations. For example, the mean-square deviation of an arbitrary quantity $f$ can be obtained directly from Eqs. (15a) and (15b), yielding up to order $\Omega^{-1}$

$$
\left\langle f^{2}(x) \mid x_{0}\right\rangle_{\tau}-\left\langle f(x) \mid x_{0}\right\rangle_{\tau}^{2}=\frac{2}{\Omega} \chi_{1,2}\left[f^{(1)}(\bar{x})\right]^{2} .
$$

This shows that fluctuations are of order $\Omega^{-1}$, and $\chi_{1,2}$ measures the size of these fluctuations. (For an explicit expression for $\chi_{1,2}$ see Sec. III.)

In the next section we will focus our attention on the general structure of $\chi_{l, 2 l-k}$ and establish a recursion relation for these functions. It will turn out that for a fixed value of $k$ the quantities $\chi_{l, 2 l-k}$ which belong to the $(k+1)$ th column of Eq. (15a) are polynomials in $\chi_{1,2}$ and the dependence on the index $l$ is explicitly given by the powers of $\chi_{1,2}$. In particular, for $k=0$ we have already shown that the terms in the first column of Eq. (15a) can be expressed by

$$
\chi_{l, 2 l}=\frac{\chi_{1,2}^{l}}{l !}
$$

which-neglecting all other terms-gives rise to a Gaussian distribution function with time-dependent mean $\bar{x}$ and variance $2 \chi_{1,2}$. This approximation is the well-known linear noise approximation. ${ }^{2}$ However, from a mathematical point of view, there is no a priori reason for neglecting all other terms in Eq. (15b), and the question arises, what are the contributions of these terms? In the following sections we will address this question and show that each column in Eq. (15b) gives rise to a Hermite function in the TDF meaning that $h\left(x, t \mid x_{0}\right)$ can be expanded in a series of Hermite functions, where the coefficients of the series can be determined successively.

\section{RECURSION RELATION FOR $\chi_{l, 2 l-k}$}

In this section we want to give both a recursion relation for the functions $\chi_{l, 2 l-k}$ and their explicit dependence on the index $l$ for a fixed value of $k$. We start combining Eqs. (9a) and (9b) to obtain

$$
\chi_{l}\left[x_{0}, \bar{x}\right]=\sum_{s=2}^{l+1} \frac{1}{s !} \int_{x_{0}}^{\bar{x}} \frac{\alpha_{s}(y)}{\alpha_{1}(y)}\left(\frac{d^{s}}{d x_{0}^{s}} \chi_{l+1-s}\left[x_{0}, \bar{x}\right]\right)_{x_{0}=y} d y, l \geq 1
$$


Since the "initial condition" $\chi_{0}=f(\bar{x})$ is known, we can use Eq. (18) to determine the $\chi_{l}$ 's successively. An explicit expression for $\chi_{1}$ has been given previously ${ }^{10}$ and shows agreement with the general structure of Eq. (14). Note that the differentiation on the rhs of Eq. (18) has to be understood according to Eq. (13), since $\bar{x}$ depends on $x_{0}$.

Let us first simplify the notation: Suppose $B\left[x_{0}, \bar{x}\right]$ is an arbitrary function of the two-independent variables $x_{0}$ and $\bar{x}$. In order to denote the $k$ th total derivative with respect to $x_{0}$ we use a superscript in square brackets and suppress the argument.

$$
\frac{d^{k}}{d x_{0}^{k}} B\left[x_{0}, \bar{x}\right]=B^{[k]} \text {. }
$$

By $f^{(n)}(\bar{x})$ we denote the $n$th derivative of $f$ with respect to the argument which therefore does not depend explicitly on $x_{0}$. Furthermore we suppress the integration variable $y$. With this simplified notation Eq. (18) reads

$$
\chi_{l}=\sum_{s=2}^{l+1} \frac{1}{s !} \int_{x_{0}}^{\bar{x}} \frac{\alpha_{s}}{\alpha_{1}} \chi l_{+1-s}^{[s]} d y, l \geq 1 .
$$

In order to find a recursion relation for the $\chi_{l, 2 l-k}$ [see Eqs. (15a) and (15b)] we first prove that the general structure of $\chi_{l}$ is given by

$$
\chi_{l}\left[x_{0}, \bar{x}\right]=\sum_{k=0}^{2 l-1} f^{(2 l-k)}(\bar{x}) \chi_{l, 2 l-k}\left[x_{0}, \bar{x}\right]
$$

with

$$
\chi_{l, 2 l-k} \equiv 0 \text { for } l \leq 0 \text { or } k<0 \text { or } k \geq 2 l \text { but } \chi_{0,0} \equiv 1,
$$

where the $\chi_{l, 2 l-k}$ 's no longer depend on $f$. We know already that $\chi_{0}$ and $\chi_{1}$ have the form of Eq. (21) and for general $l \geq 1$ we will prove this relation by induction. We start assuming that Eq. (21) is valid for all $\chi_{L}$ with $0 \leq L \leq l-1$. Since on the rhs of Eq. (20) we have only derivatives of $\chi_{L}$ with $0 \leq L \leq l-1$, we can insert our assumption [Eq. (21)] into Eq. (20) in order to prove that the left-hand side (lhs) of Eq. (20) has also the assumed form. In addition this procedure will provide us with a recursion relation for $\chi_{l, 2 l-k}$. First we have to differentiate Eq. (21) with respect to $x_{0}$

$$
\begin{aligned}
\chi_{L}^{[s]} & =\sum_{k=0}^{2 L-1} \sum_{m=0}^{s}\left[\begin{array}{c}
s \\
m
\end{array}\right]\left[\frac{d^{m}}{d x_{0}^{m}} f^{(2 L-k)}(\bar{x})\right] \chi_{L, 2 L-k}^{s-m]} \\
& =\sum_{k=0}^{2 L-1} \sum_{m=0}^{s} \sum_{j=0}^{m}\left[\begin{array}{c}
s \\
m
\end{array}\right] f^{(2 L-k+j)}(\bar{x}) p_{m, j} \chi_{L, 2 L-k}^{[s-m]},
\end{aligned}
$$

where we have used Leibnitz' rule in the first step and the chain rule for higher derivatives in the second step (see Appendix A). Note that in Eq. (23) the dependence on $f$ is explicit and $p_{m, j}=p_{m, j}\left[x_{0}, \bar{x}\right]$. If we now rearrange the sums in Eq. (23) with respect to the derivatives of $f$ we obtain (see Appendix C)

$$
\begin{aligned}
\chi_{L}^{[s]}=\sum_{k=0}^{2 L}+s-1 & f^{(2 L+s-k)}(\bar{x}) \\
& \times \sum_{j=0}^{s} \sum_{m=j}^{s}\left[\begin{array}{c}
s \\
m
\end{array}\right]_{m, j} \chi_{L, 2 L+s-j-k}^{[s-m]},
\end{aligned}
$$

where for $\chi_{l, 2 l-k}$ the constraints, Eq. (22), have to be used.

Putting $L=l+1-s$ and inserting this expression into the rhs of Eq. (20) we obtain-again after rearranging the series with respect to the derivatives of $f$ -

$$
\chi_{l}=\sum_{k=0}^{2 l-1} f^{(2 l-k)}(\bar{x}) \sum_{j=2}^{k+2} \sum_{m=0}^{j} \sum_{n=m}^{j} \frac{1}{j !}\left[\begin{array}{l}
j \\
n
\end{array}\right) \int_{x_{0}}^{\bar{x}} \frac{\alpha_{j}}{\alpha_{1}} p_{n, m} \chi l_{+1-j, 2 l-k-m}^{j-n]} d y, \quad l \geq 1
$$

which proves Eq. (21), since Eq. (25) has the same structure. In addition, comparing Eq. (25) with Eq. (21), we immediately obtain the following recursion relation for $\chi_{l, 2 l-k}$ :

$$
\chi_{l, 2 l-k}=\sum_{j=2}^{k+2} \sum_{m=0}^{j} \sum_{n=m}^{j} \frac{1}{j !}\left[\begin{array}{l}
j \\
n
\end{array}\right] \int_{x_{0}}^{\bar{x}} \frac{\alpha_{j}}{\alpha_{1}} p_{n, m} \chi l_{+1-j, 2 l-k-m}^{j-n]} d y, \quad l \geq 1
$$

which enables us to calculate the functions $\chi_{l, 2 l-k}$ successively. In order to see this more clearly we change the indices $(j, m, n)$ to new indices $(L, K, J)$ via the relations

$$
L=l+1-j, \quad K=k+m+2-2 j, \quad J=j-n .
$$

Equation (26) then assumes the form

$$
\chi_{l, 2 l-k}=\sum_{(L, K, J) \in \mathscr{S}_{\mathrm{I}}} \int_{x_{0}}^{\bar{x}} \alpha_{L, K, J}^{l, 2 l-k} \chi_{L, 2 L-K}^{[J]} d y
$$

The functions $\alpha_{L, K, J}^{l, 2 l-k}$, which depend on $x_{0}$ and $\bar{x}$, are given by

$$
\begin{aligned}
\alpha_{L, K, J}^{l, 2 l-k}= & \frac{1}{(l+1-L) !}\left[\begin{array}{c}
l+1-L \\
l+1-L-J
\end{array}\right] \\
& \times \frac{\alpha_{l+1-L}}{\alpha_{1}} p_{l+1-L-J, 2 l-k-2 L+K}
\end{aligned}
$$

and the summation runs over all integer triples $(L, K, J)$ of the set $\mathscr{S}_{\mathrm{I}}$, which is defined by

$$
\begin{aligned}
\mathscr{S}_{\mathrm{I}} \equiv\{ & (L, K, J) \in \mathbb{Z}^{3} \mid \\
& l-k-1 \leq L \leq l-1, \\
& k+2 L-2 l \leq K \leq k-l+L+1, \\
& 0 \leq J \leq L-K-l+k+1\} .
\end{aligned}
$$


The inequalities, Eqs. (30a)-(30c), are immediate consequences of the index transformation Eq. (27). We want to mention once again that in evaluating the summation on the rhs of Eq. (28) the constraints given in Eq. (22) have to be taken into account.

Next we want to show that the sum on the rhs of Eq. (28) contains only one term with $K=k$. From Eqs. (30a) and (30b) we obtain $K \leq k-l+L+1 \leq k$, where the equal sign holds only for $L=l-1$. That is to say, for $L<l-1$ we cannot find a value of $K$ with $K=k$. Inserting now $(L, K)=(l-1, k)$ into Eq. (30c) yields $J=0$. We conclude that $(L, K, J)=(l-1, k, 0)$ is the only triple for which $K=k$. The corresponding value for $\alpha_{L, K, J}^{l, 2 l-k}$ is easily found to be

$$
\alpha_{l-1, k, 0}^{l, 2 l-k}=\frac{1}{2} \frac{\alpha_{2}}{\alpha_{1}} p_{2,2}=\frac{1}{2} \frac{\alpha_{2}}{\alpha_{1}}\left(\bar{x}^{[1]}\right)^{2}
$$

We can now split up the sum in Eq. (28) into two terms, one with $K=k$ and the other with $K<k$ yielding

$$
\begin{aligned}
\chi_{l, 2 l-k}= & \frac{1}{2} \int_{x_{0}}^{\bar{x}} \frac{\alpha_{2}}{\alpha_{1}}\left(\bar{x}^{[1]}\right)^{2} \chi_{l-1,2(l-1)-k} d y \\
& +\sum_{(L, K, J) \in \mathscr{S}_{\mathrm{II}}} \int_{x_{0}}^{\bar{x}} \alpha_{L, K, J}^{l, 2 l-k} \chi_{L, 2 L-K}^{[J]} d y,
\end{aligned}
$$

where $\mathscr{S}_{\text {II }}$ is the region $\mathscr{S}_{\mathrm{I}}$ minus the point $(L, K, J)=(l-1, k, 0)$, i.e., $\mathscr{S}_{\mathrm{II}}=\mathscr{S}_{\mathrm{I}} \backslash\{(l-1, k, 0)\}$. We now evaluate the recursion relation, Eq. (32), for $k=0$. The sum in Eq. (32) is zero, since for $k=0$ the set $\mathscr{S}_{\mathrm{II}}$ is empty. Therefore we get

$$
\chi_{l, 2 l}=\frac{1}{2} \int_{x_{0}}^{\bar{x}} \frac{\alpha_{2}}{\alpha_{1}}\left(\bar{x}^{[1]}\right)^{2} \chi_{l-1,2 l-2} d y .
$$

This recursion relation can be solved explicitly, since the initial value $\chi_{0,0} \equiv 1$ is known. For $l=1$ we get with the aid of Eq. (12)

$\chi_{1,2}\left[x_{0}, \bar{x}\right]=\frac{1}{2} \int_{x_{0}}^{\bar{x}} \frac{\alpha_{2}}{\alpha_{1}}\left(\bar{x}^{[1]}\right)^{2} d y=\frac{1}{2} \alpha_{1}^{2}(\bar{x}) \int_{x_{0}}^{\bar{x}} \frac{\alpha_{2}(y)}{\alpha_{1}^{3}(y)} d y$.

Introducing the abbreviation

$$
\chi_{1,2}^{\prime}:=\frac{\partial}{\partial y} \chi_{1,2}[y, \bar{x}]
$$

we can rewrite Eq. (33) in the form

$$
\chi_{l, 2 l}=-\int_{x_{0}}^{\bar{x}} \chi_{1,2}^{\prime} \chi_{l-1,2 l-2} d y
$$

or explicitly

$$
\chi_{l, 2 l}=\frac{\chi_{1,2}^{l}}{l !}
$$

Equation (37) can be easily proved by induction and was obtained previously. ${ }^{10}$ For convenience we define $\chi_{1,2}^{l} \equiv 0$ for $l<0$ in order to declare Eq. (37) valid for all $l$. This is in agreement with Eq. (24) and shall be tacitly assumed in the sequel.

Next let us turn to the case $k=1$. From Eqs. (30a) and (30b) we get for the region $\mathscr{S}_{\text {II }} K=0$; therefore the sum on the rhs of Eq. (32) contains only terms of the form $\chi_{L, 2 L}^{[J]}$, the explicit structure of which is already known for all $L$ due to Eq. (37). In particular, region $\mathscr{S}_{\text {II }}$ consists of the following set of triples:

$$
\mathscr{S}_{\mathrm{II}}=\{(l-1,0,1),(l-1,0,0),(l-2,0,0)\}
$$

and the corresponding values for $\alpha_{L, K, J}^{l, 2 l-k}$ are given by [see Eq. (29) and Appendix A]

$$
\begin{aligned}
& \alpha_{l-1,0,1}^{l, 2 l-1}=\frac{\alpha_{2}}{\alpha_{1}} \bar{x}^{[1]}, \\
& \alpha_{l-1,0,0}^{l, 2 l-1}=\frac{1}{2} \frac{\alpha_{2}}{\alpha_{1}} \bar{x}^{[2]}, \\
& \alpha_{l-2,0,0}^{l, 2 l-1}=\frac{1}{6} \frac{\alpha_{3}}{\alpha_{1}}\left(\bar{x}^{[1]}\right)^{3} .
\end{aligned}
$$

Therefore Eq. (32) reduces for $k=1$ to

$$
\begin{aligned}
\chi_{l, 2 l-1}= & \left.-\int_{x_{0}}^{\bar{x}} \chi_{1,2}^{\prime} \chi_{l-1,2 l-3} d y+\int_{x_{0}}^{\bar{x}} \frac{\alpha_{2}}{\alpha_{1}} \bar{x}^{[1]} \chi\right\}_{-1,2 l-2}^{[1]} d y \\
& +\frac{1}{2} \int_{x_{0}}^{\bar{x}} \frac{\alpha_{2}}{\alpha_{1}} \bar{x}^{[2]} \chi_{l-1,2 l-2} d y \\
& +\frac{1}{6} \int_{x_{0}}^{\bar{x}} \frac{\alpha_{3}}{\alpha_{1}}\left(\bar{x}^{[1]}\right)^{3} \chi_{l-2,2 l-4} d y
\end{aligned}
$$

Inserting now the explicit expression for $\chi_{l, 2 l}$ given by Eq. (37) yields

$$
\begin{aligned}
\chi_{l, 2 l-1}= & -\int_{x_{0}}^{\bar{x}} \chi_{1,2}^{\prime} \chi_{l-1,2 l-3} \\
& +\int_{x_{0}}^{\bar{x}}\left[\frac{1}{2} \frac{\alpha_{2}}{\alpha_{1}} \bar{x}^{[2]}\right] \frac{\chi_{1,2}^{l-1}}{(l-1) !} d y \\
& +\int_{x_{0}}^{\bar{x}}\left(\frac{\alpha_{2}}{\alpha_{1}} \bar{x}^{[1]} \chi_{1,2}^{[1]}+\frac{1}{6} \frac{\alpha_{3}}{\alpha_{1}}\left(\bar{x}^{[1]}\right)^{3}\right) \frac{\chi_{1,2}^{l-2}}{(l-2) !} d y .
\end{aligned}
$$

Equation (41) is now a recursion relation in $l$ alone and can be solved explicitly using Eq. (B2) of Appendix B. The result is

$$
\chi_{l, 2 l-1}=\bar{\chi}_{1,1} \frac{\chi_{1,2}^{l-1}}{(l-1) !}+\bar{\chi}_{2,3} \frac{\chi_{1,2}^{l-2}}{(l-2) !}
$$

with

$$
\bar{\chi}_{1,1}=\frac{1}{2} \int_{x_{0}}^{\bar{x}} \frac{\alpha_{2}}{\alpha_{1}} \bar{x}^{[2]} d y
$$

and

$$
\bar{\chi}_{2,3}=\int_{x_{0}}^{\bar{x}} \frac{\alpha_{2}}{\alpha_{1}} \bar{x}^{[1]} \chi_{1,2}^{[1]} d y+\frac{1}{6} \int_{x_{0}}^{\bar{x}} \frac{\alpha_{3}}{\alpha_{1}}\left(\bar{x}^{[1]}\right)^{3} d y .
$$

Again we have to keep in mind that by definition $\chi_{1,2}^{l} \equiv 0$ for $l<0$. Equation (42) implies a considerable simplification of our scheme, Eq. (15b), since once the functions $\bar{\chi}_{1,1}$ and $\bar{\chi}_{2,3}$ are calculated, we know all terms in the second column of Eq. (15b).

It is now obvious how the functions $\chi_{l, 2 l-2}$ can be calculated. For region $\mathscr{S}_{\text {II }}$ we now have $K=0,1$. This im- 
plies that on the rhs of Eq. (32) we have only terms of the form $\chi_{L, 2 L}^{[J]}$ and $\chi_{L, 2 L-1}^{[J]}$, the structure of which is already known for all $L$ [see Eqs. (37) and (42)]. Since the considerations are identical to those for $\chi_{l, 2 l-1}$, we only present the result. The functions $\chi_{l, 2 l-2}$ are again polynomials in $\chi_{1,2}$ and have the form

$$
\begin{aligned}
\chi_{l, 2 l-2}= & \left(\frac{1}{2} \bar{\chi}_{1,1}^{2}+\bar{\chi}_{2,2}\right) \frac{\chi_{1,2}^{l-2}}{(l-2) !}+\left(\bar{\chi}_{1,1} \bar{\chi}_{2,3}+\bar{\chi}_{3,4}\right) \frac{\chi_{1,2}^{l-3}}{(l-3) !} \\
& +\frac{1}{2} \bar{\chi}_{2,3}^{2} \frac{\chi_{1,2}^{l-4}}{(l-4) !}
\end{aligned}
$$

where the new coefficients $\bar{\chi}_{2,2}$ and $\bar{\chi}_{3,4}$ are given by

$$
\begin{aligned}
\bar{\chi}_{2,2}= & \frac{1}{2} \int_{x_{0}}^{\bar{x}} \frac{\alpha_{2}}{\alpha_{1}} \chi_{1,2}^{[2]} d y+\int_{x_{0}}^{\bar{x}} \frac{\alpha_{2}}{\alpha_{1}} \bar{x}^{[1]} \chi_{1,1}^{[1]} d y \\
& +\frac{1}{2} \int_{x_{0}}^{\bar{x}} \frac{\alpha_{3}}{\alpha_{1}} \bar{x}^{[1]} \bar{x}^{[2]} d y
\end{aligned}
$$

and

$$
\begin{aligned}
\bar{\chi}_{3,4}= & \frac{1}{2} \int_{x_{0}}^{\bar{x}} \frac{\alpha_{2}}{\alpha_{1}}\left(\chi_{1,2}^{[1]}\right)^{2} d y+\int_{x_{0}}^{\bar{x}} \frac{\alpha_{2}}{\alpha_{1}} \bar{x}^{[1]} \bar{\chi}_{2,3}^{[1]} d y \\
& +\frac{1}{2} \int_{x_{0}}^{\bar{x}} \frac{\alpha_{3}}{\alpha_{1}}\left(\bar{x}^{[1]}\right)^{2} \chi_{1,2}^{[1]} d y+\frac{1}{24} \int_{x_{0}}^{\bar{x}} \frac{\alpha_{4}}{\alpha_{1}}\left(\bar{x}^{[1]}\right)^{4} d y
\end{aligned}
$$

Although the coefficients $\bar{\chi}_{2,2}$ and $\bar{\chi}_{3,4}$ are rather involved, we see that the structure of $\chi_{l, 2 l-2}$ remains simple. Equation (45) represents an analytic expression for the coefficients in the third column of Eq. (15a). In principle no difficulty arises when calculating the terms $\chi_{l, 2 l-k}$ with $k \geq 3$. However, rather than going beyond our present explicit calculations, we want to focus our attention on the general structure of $\chi_{l, 2 l-k}$. In the next section we shall prove that for all $k$ the dependence of $\chi_{l, 2 l-k}$ on the index $l$ is given by a polynomial in $\chi_{1,2}$. Furthermore the upper and lower index of this polynomial are given. It is exactly this explicit structure of $\chi_{l, 2 l-k}$ which will enable us to reconstruct the form of the distribution function itself. This will be done in Sec. V.

\section{GENERAL STRUCTURE OF $\chi_{l, 2 l-k}$}

According to the results of the previous section it seems quite obvious that the dependence of $\chi_{l, 2 l-k}$ on the index $l$ is given by a polynomial in $\chi_{1,2}$. However, the determination of the upper and lower index of this polynomial is not evident. Therefore we want to prove in this section that the general structure of $\chi_{L, 2 L-K}$ is given by

$$
\chi_{L, 2 L-K}=\sum_{I=[K / 2]+1}^{2 K} c_{K, I} \frac{\chi_{1,2}^{L-I}}{(L-I) !}, K \geq 1,
$$

where $c_{K, I}$ are functions of $x_{0}$ and $\bar{x}$ but independent of $L$. They can be determined uniquely by solving the recursion relation Eq. (28). In particular, by comparing Eq. (48) with Eq.s (42) and (45) we get for the first few $c_{K, I}$

$c_{1,1}=\bar{\chi}_{1,1}, \quad c_{1,2}=\bar{\chi}_{2,3}$, $c_{2,2}=\frac{\bar{\chi}_{1,1}^{2}}{2}+\bar{\chi}_{2,2}, \quad c_{2,3}=\bar{\chi}_{1,1} \bar{\chi}_{2,3}+\bar{\chi}_{3,4}, \quad c_{2,4}=\frac{\bar{\chi}_{2,3}^{2}}{2}$

In Eq. (48) the symbol $[K / 2]$ denotes the largest integer less or equal to $K / 2$, and $\chi_{1,2}^{L} \equiv 0$ for $L<0$, as defined in Sec. III. It should be stressed that Eq. (48) holds for $K \geq 1$ only, and in the case $K=0$ we have to use Eq. (37). Taking now the $J$ th total derivative with respect to $x_{0}$ of both Eq. (48) and Eq. (37) we get

$\chi_{L, 2 L-K}^{[J]}=\sum_{I=[K / 2]+1}^{2 K+J} c_{K, I}^{J} \frac{\chi_{1,2}^{L-I}}{(L-I) !}$, for $K, J=0,1,2, \ldots$

but $(K, J) \neq(0,0)$ and

$$
\chi_{L, 2 L-K}^{[J]}=\frac{\chi_{1,2}^{L}}{L !} \text { for }(K, J)=(0,0)
$$

and the coefficients $c_{K, I}^{J}$ are uniquely determined by this differentiation.

According to Eqs. (51a) and (51b) let us next split up the sum in Eq. (32) into two terms, one with $(K, J)=(0,0)$ and the other with $(K, J) \neq(0,0)$

$$
\begin{aligned}
\chi_{l, 2 l-k}= & -\int_{x_{0}}^{\bar{x}} \chi_{1,2}^{\prime} \chi_{l-1,2 l-2-k} d y \\
& +\sum_{\substack{(L, K, J) \in \mathscr{S}_{\mathrm{II}} \\
(K, J)=(0,0)}} \int_{x_{0}}^{\bar{x}} \alpha_{L, 0,0}^{l, 2 l-k} \chi_{L, 2 L} d y \\
& +\sum_{\substack{(L, K, J) \in \mathscr{S}_{\mathrm{II}} \\
(K, J) \neq(0,0)}} \int_{x_{0}}^{\bar{x}} \alpha_{L, K, J}^{l, 2 l-k} \chi_{L, 2 L-K}^{[J]} d y .
\end{aligned}
$$

Note that for $k=0$ both sums are empty. In order to prove Eq. (48) we assume that Eq. (48) [and Eqs. (51a) and (51b) which are a consequence of Eq. (48)] is valid for all $K \leq k-1$ and for all $l$. In the case of $K=0,1,2$ this has been shown explicitly in Sec. III. Since on the rhs of Eq. (52) we only have terms $\chi_{L, 2 L-K}^{[J]}$ with $K \leq k-1$, we can insert our assumption Eqs. (51a) and (51b) in order to prove that $\chi_{l, 2 l-k}$ has again the structure given by Eq. (48). In the following we consider each sum in Eq. (52) separately.

(i) Inserting Eq. (51b) into Eq. (52) we obtain for the first sum

$$
A:=\sum_{\substack{(L, K, J) \in \mathscr{S}_{\mathrm{II}} \\(K, J)=(0,0)}} \int_{x_{0}}^{\bar{x}} \alpha_{L, 0,0}^{l, 2 l-k} \frac{\chi_{1,2}^{L}}{L !} d y,
$$

where the sum has to be taken over the subregion of $\mathscr{S}_{\mathrm{II}}$ defined by $(K, J)=(0,0)$. Putting $(K, J)=(0,0)$ in Eqs. (30a) and (30b) we find the following inequalities for $L$ :

$$
\begin{aligned}
& l-k-1 \leq L \leq l-1, \\
& 2 L \leq 2 l-k .
\end{aligned}
$$

The coefficients $\alpha_{L, 0,0}^{l, 2 l-k}$ are given by [see Eq. (29)]

$$
\alpha_{L, 0,0}^{l, 2 l-k}=\frac{1}{(l+1-L) !} \frac{\alpha_{l+1-L}}{\alpha_{1}} p_{l+1-L, 2 l-k-2 L} .
$$


If we now keep in mind that $p_{n, 0}=\delta_{n, 0}$ (see Appendix A), we see that, if $2 L=2 l-k$ [which is possible due to Eq. (54b)], the corresponding coefficient $\alpha_{L, 0,0}^{l, 2 l-k}$ is proportional to $p_{l+1-L, 0}$, which is zero unless $L=l+1$. But $L=l+1$ is in contradiction to Eq. (54a). We therefore can write instead of Eq. (54b) the inequality $2 L<2 l-k$, which is equivalent to $L \leq l-[k / 2]-1$, since $l$ has to be an integer. This latter inequality is for $k \geq 0$ always stronger than Eq. (54a) and so we can replace Eqs. (54a) and $(54 \mathrm{~b})$ by

$$
l-k-1 \leq L \leq l-[k / 2]-1 .
$$

Performing the index transformation $L=l-i$ in Eq. (53) we get

$$
A=\sum_{i=[k / 2]+1}^{k+1} \int_{x_{0}}^{\bar{x}} \alpha_{l-i, 0,0}^{l, 2 l-k} \frac{\chi_{1,2}^{l-i}}{(l-i) !} d y .
$$

According to the remark following Eq. (52) the above expression is only valid for $k \geq 1$. Defining now coefficients $A_{i, k}$ as follows:

$$
A_{i, k}:=\left\{\begin{array}{lr}
\alpha_{l-i, 0,0}^{l, 2 l-k}=\frac{1}{(i+1) !} \frac{\alpha_{i+1}}{\alpha_{1}} p_{i+1,2 i-k} \\
0, \text { for } i>k+1 . & \text { for } i \leq k+1,
\end{array}\right.
$$

We can extend the upper index of the sum in Eq. (57) to $2 k$ yielding

$$
A=\sum_{i=[k / 2]+1}^{2 k} \int_{x_{0}}^{\bar{x}} A_{i, k} \frac{\chi_{1,2}^{l-i}}{(l-1) !} d y
$$

where it should be noted that according to Eq. (58a) and (58b) the coefficients $A_{i, k}$ are independent of $l$.

(ii) Inserting Eq. (51a) into the second sum of Eq. (52) we obtain

$$
B:=\sum_{\substack{(L, K, J) \in \mathscr{S}_{\mathrm{II}} \\(K, J) \neq(0,0)}} \sum_{I=[K / 2]+1}^{2 K+J} \int_{x_{0}}^{\bar{x}} c_{K, I}^{J} \alpha_{L, K, J}^{l, 2 l-k} \frac{\chi_{1,2}^{L}-I}{(L-I) !} d y,
$$

where the summation runs over all triples $(L, K, J)$ from region $\mathscr{S}_{\text {II }}$ excluding the points with $(K, J)=(0,0)$, i.e., over all $(L, K, J) \in \mathscr{S}_{\mathrm{II}} \backslash\left\{(L, K, J) \in \mathscr{S}_{\mathrm{II}} \mid K=J=0\right\}$. Performing an index transformation $(L, K, J, I)$ $\rightarrow(l-L, K, J, I)$ we obtain

$$
B=\sum_{(L, K, J, I) \in \mathscr{S}_{\mathrm{III}}} \int_{x_{0}}^{\bar{x}} c_{K, I}^{J} \alpha_{l-L, K, J}^{l, 2 l-k} \frac{\chi_{1,2}^{l-L-I}}{(l-L-I) !} d y,
$$

where the sum has to be taken over all integer quadruples $(L, K, J, I) \in \mathscr{S}_{\mathrm{III}}$, given by

$$
\begin{aligned}
& \mathscr{S}_{\mathrm{III}} \equiv\left\{(L, K, J, I) \in Z^{4} \mid(L, K, J, I) \neq(1, k, 0, I)\right. \\
& 1 \leq L \leq k+1 \\
& k-2 L \leq K \leq k-L+1
\end{aligned}
$$

$$
\begin{aligned}
& 0 \leq J \leq k+1-K-L, \\
& [K / 2]+1 \leq I \leq 2 K+J\} .
\end{aligned}
$$

The coefficients $\alpha_{l-L, K, J}^{l, 2 l-k}$ are given by [see Eq. (29)]

$$
\alpha_{l-L, K, J}^{l, 2 l-k}=\frac{1}{(L+1) !}\left(\begin{array}{c}
L+1 \\
L+1-j
\end{array}\right) \frac{\alpha_{L+1}}{\alpha_{1}} p_{L+1-j, K-k+2 L}
$$

and are independent of the index $l$ since neither Eqs. (62a)-(62d) nor the rhs of Eq. (63) depend on $l$. Next we want to prove that Eq. (61) can be written in the same form as Eq. (59), namely

$$
B=\sum_{i=[k / 2]+1}^{2 k} \int_{x_{0}}^{\bar{x}} B_{i, k} \frac{\chi_{1,2}^{l-i}}{(l-i) !} d y .
$$

The proof will be completed, if we can show that $I+L$ in Eq. (61) satisfies the inequality

$$
[k / 2]+1 \leq I+L \leq 2 k
$$

because then we can rearrange Eq. (61) according to powers of $\chi_{1,2}$ and arrive at Eq. (64). To this end we first insert the lhs of Eq. (62b) into the lhs of Eq. (62d) and get

$$
\begin{aligned}
& I \geq\left[\frac{K}{2}\right]+1 \geq\left[\frac{k-2 L}{2}\right]+1=\left[\frac{k}{2}\right]+1-L \\
& \text { or } \quad I+L \geq\left[\frac{k}{2}\right]+1
\end{aligned}
$$

which we wanted to prove. Next we note that Eqs. (62c) and (62d) imply

$$
I \leq k+1+K-L \text { or } I+L \leq k+1+K .
$$

For $L=1$ we have $K \leq k-1$ because of $(L, K, J) \neq(1, k, 0)$ and therefore $I+L \leq 2 k$; for $L \geq 2$ we have $K \leq k-1$ from Eq. (62b) and therefore again $I+L \leq 2 k$. So we have shown that Eq. (65) is valid and therefore the expression given in Eq. (64) exists.

Inserting now Eq. (64) and (59) into Eq. (52) we obtain the recursion relation

$$
\begin{aligned}
\chi_{l, 2 l-k}= & -\int_{x_{0}}^{\bar{x}} \chi_{1,2} \chi_{l-1,2 l-2-k}^{\prime} d y \\
& +\sum_{i=[k / 2]+1}^{2 k} \int_{\bar{x}_{0}}^{\bar{x}} C_{i, k} \frac{\chi_{1,2}^{l-i}}{(l-i) !} d y
\end{aligned}
$$

with $C_{i, k}:=A_{i, k}+B_{i, k}$. Note that Eq. (66) is a recursion relation in $l$ alone with a fixed index $k$, since it has been derived under the assumption that $\chi_{L, 2 L-K}$ has the form of Eq. (48) for $K \leq k-1$ and all $l$. The explicit solution of Eq. (66) can be found easily, if we make use of lemma 1 given in Appendix B. We obtain the relation

$$
\chi_{l, 2 l-k}=\sum_{i=[k / 2]+1}^{2 k} \frac{\chi_{1,2}^{l-i}}{(l-1) !} \int_{x_{0}}^{\bar{x}} C_{i, k} d y
$$

which is exactly the form of Eq. (48) and completes our proof. 
We briefly want to recall the significance of our result derived in this section. We have shown that the dependence of the functions $\chi_{l, 2 l-k}$ on the index $l$ is explicitly given by a polynomial in $\chi_{1,2}$. The coefficients $c_{i, k}$ of this polynomial can be determined successively by solving Eq. (32). We therefore have found analytic expressions for the coefficients in each column of Eq. (15a). In the next section we will see how this information can be used to derive the distribution function itself.

\section{EXPLICIT FORM OF THE DISTRIBUTION FUNCTION}

We have already shown in a previous paper ${ }^{10}$ that the terms in the first column of Eq. (15a) give rise to a Gaussian distribution function. This approximation was called linear noise approximation. If this approximation is used for calculating conditional averages $\chi\left(x_{0}, \tau\right)$ we see from Eq. (15b) that the linear noise approximation does not provide a systematic expansion of $\chi$ in powers of $\Omega^{-1}$. To see this more clearly, let us consider the special conditional average $\left\langle x \mid x_{0}\right\rangle_{\tau}$. From Eqs. (15b) and (48) we find immediately

$$
\left\langle x \mid x_{0}\right\rangle_{\tau}=\bar{x}+\sum_{l=1}^{\infty} \frac{1}{\Omega^{l}} \chi_{l, 1}=\bar{x}+\sum_{l=1}^{\infty} \frac{c_{2 l-1, l}}{\Omega^{l}},
$$

whereas the linear noise approximation yields only the first term on the rhs of Eq. (68). One can argue that in many examples the parameter $\Omega$ is very large and contributions of order $\Omega^{-1}$ can be neglected. To be consistent, however, one should then neglect all terms of order $\Omega^{-1}$ in Eq. (15b), which implies for the distribution function $h\left(x, t \mid x_{0}\right)=\delta(x-\bar{x})$. This, however, describes only the deterministic (macroscopic) motion of the system and gives no information about fluctuations, which are of or$\operatorname{der} \Omega^{-1}$ [see Eq. (16)].

Another argument for neglecting the sum on the rhs of Eq. (68) could be the following. Suppose that the functions $\chi_{l, 1}$ are small compared to the macroscopic motion $\bar{x}$ of the system. Then we could neglect them even for a finite value of $\Omega^{-1}$. But this assumption, namely $\chi_{l, 1}<<\bar{x}$, is in general not true. However, in contrast to our arguments it is known that the linear noise approximation provides a satisfactory description of the stochastic process, if the parameter $\Omega$ is large. The purpose of this section is to resolve this "discrepancy" and to give a mathematical limit for which the linear noise approximation holds. Furthermore we want to present corrections to the linear noise approximation, which are necessary, if the parameter $\Omega$ in the physical system under considerations is not "infinitely large."

Let us start by combining Eq. (6) with Eq. (14). We obtain for the conditional average of an arbitrary quantity $f$ [see also Eq. (15b)]

$$
\begin{aligned}
\left\langle f(x) \mid x_{0}\right\rangle_{\tau}= & \sum_{l=0}^{\infty} \frac{1}{\Omega^{l}} f^{(2 l)}(\bar{x}) \frac{\chi_{1,2}^{l}}{l !} \\
& +\sum_{l=1}^{\infty} \frac{1}{\Omega^{l}} \sum_{k=1}^{2 l-1} f^{(2 l-k)}(\bar{x}) \chi_{l, 2 l-k} .
\end{aligned}
$$

Next we rearrange the second sum on the rhs of Eq. (69) performing first the summation in each column of Eqs. (15a) and (15b) and afterwards the summation with respect to the rows. One easily finds

$$
\begin{aligned}
\left\langle f(x) \mid x_{0}\right\rangle_{\tau}= & \sum_{l=0}^{\infty} \frac{1}{\Omega^{l}} f^{(2 l)}(\bar{x}) \frac{\chi_{1,2}^{l}}{l !} \\
& +\sum_{k=1}^{\infty} \sum_{l=[k / 2]+1}^{\infty} \frac{1}{\Omega^{l}} f^{(2 l-k)}(\bar{x}) \chi_{l, 2 l-k} .
\end{aligned}
$$

In what follows we consider only the second sum on the rhs of Eq. (70). Denoting this expression with the letter $A$ we obtain after inserting our explicit expression for $\chi_{l, 2 l-k}$ [cf. Eq. (48)]

$$
\begin{aligned}
A & :=\sum_{k=1}^{\infty} \sum_{i=[k / 2]+1}^{2 k} c_{k, i} \sum_{l=i}^{\infty} \frac{1}{\Omega^{l}} f^{(2 l-k)}(\bar{x}) \frac{\chi_{1,2}^{l-i}}{(l-i) !} \\
& =\sum_{k=1}^{\infty} \sum_{i=[k / 2]+1}^{2 k} c_{k, i} \sum_{l=0}^{\infty} \frac{1}{\Omega^{l+i}} f^{(2 l+2 i-k)}(\bar{x}) \frac{\chi_{1,2}^{l}}{l !} .
\end{aligned}
$$

In the first expression we made use of our definition $\chi_{1,2}^{k} \equiv 0$ for $k<0$ leading to $\sum_{l=i}^{\infty}$ in the second one we performed an index transformation $l-i \rightarrow l$. Now the summation over $l$ is independent of all other indices and we can write

$$
A=\sum_{l=0}^{\infty} \frac{1}{\Omega^{l}} \frac{\chi_{1,2}^{l}}{l !}\left(\sum_{k=1}^{\infty} \sum_{i=[k / 2]+1}^{2 k} \frac{c_{k, i}}{\Omega^{i}} f^{(2 l+2 i-k)}(\bar{x})\right) \text {. }
$$

Finally we rearrange the term in the large parentheses with respect to derivatives of $f$

$$
\sum_{k=1}^{\infty} \sum_{i=[k / 2]+1}^{2 k} \frac{c_{k, i}}{\Omega^{i}} f^{(2 l+2 i-k)}(\bar{x})=\sum_{s=1}^{\infty} f^{(2 l+s)}(\bar{x}) d_{s} .
$$

The coefficients $d_{s}$ can be determined with the method described in Appendix C. One gets

$$
d_{s}=\sum_{i=[(2 s+2) / 3]}^{\infty} \frac{c_{2 i-s, i}}{\Omega^{i}}, d_{0}:=1
$$

and therefore

$$
A=\sum_{l=0}^{\infty} \frac{1}{\Omega^{l}} \frac{\chi_{1,2}^{l}}{l !} \sum_{s=1}^{\infty} f^{(2 l+s)}(\bar{x}) d_{s} .
$$

Inserting this result into Eq. (70) and using the explicit form of $\chi_{l, 2 l}$ [see Eq. (37)] yields

$$
\begin{aligned}
\left\langle f(x) \mid x_{0}\right\rangle_{\tau} & \equiv \int f(x) h\left(x, t \mid x_{0}\right) d x \\
& =\sum_{l=0}^{\infty} \frac{1}{l !}\left[\frac{\chi_{1,2}}{\Omega}\right]^{l} \sum_{s=0}^{\infty} d_{s} f^{(2 l+s)}(\bar{x}),
\end{aligned}
$$

where we used the definition $d_{0}:=1$. The special form of the sum in Eq. (76) allows us to apply lemma 2, Appendix B, Eq. (B3), and gives an explicit expression for the distribution function itself 


$$
\begin{aligned}
h\left(x, t \mid x_{0}\right)=\sum_{n=0}^{\infty} & {\left[\frac{\Omega}{4 \pi \chi_{1,2}}\right]^{1 / 2} \exp \left[-\Omega(x-\bar{x})^{2} / 4 \chi_{1,2}\right] } \\
& \times d_{n}\left(\frac{\Omega}{4 \chi_{1,2}}\right]^{n / 2} H e_{n}\left[\left[\frac{\Omega}{4 \chi_{1,2}}\right]^{1 / 2}(x-\bar{x})\right]
\end{aligned}
$$

This expression can be written in a simpler form, if we introduce a new stochastic variable $y(t)$ via

$$
y:=\left(\frac{\Omega}{4 \chi_{1,2}}\right)^{1 / 2}(x-\bar{x})
$$

and the distribution function $\bar{h}(y, \tau)$

$$
h\left(x, t \mid x_{0}\right) d x=: \bar{h}(y, \tau) d y \text {. }
$$

Furthermore, by expressing Eq. (77) in terms of Hermite functions $^{13}$

$$
\Psi_{n}(y)=e^{-\left(y^{2} / 2\right)} \mathrm{He}_{n}(y)
$$

one easily finds

$$
\begin{aligned}
& \bar{h}(y, \tau)=\sum_{n=0}^{\infty} b_{n} \Psi_{0}(y) \Psi_{n}(y) \\
& b_{0}:=\frac{1}{\sqrt{\pi}}, \\
& b_{n}:=\frac{1}{\sqrt{\pi}}\left(\frac{\Omega}{4 \chi_{1,2}}\right)^{n / 2} \sum_{i=[(2 n+2) / 3]}^{\infty} \frac{c_{2 i-n, i}}{\Omega^{i}}, n \geq 1 .
\end{aligned}
$$

Equation (81a) is the central result of this paper and represents the Green's function solution of the original master equation [Eq. (1a)]. It is not a formal expansion of the distribution function $\bar{h}(y, \tau)$ in Hermite functions, since the coefficients $b_{n}$ are determined by recursion relations [see Eqs. (28) and (48)] and their dependence on $\Omega$ appears explicitly. In general the coefficients $b_{n}(n \geq 1)$ depend on $x_{0}$ and on the scaled time $\tau$ via the solution of the macroscopic equation [Eq. (8)]. From the definition of the $b_{n}$ 's [Eq. (81b)] we find

$$
\lim _{\Omega \rightarrow \infty} b_{n}=b_{0} \delta_{n, 0}
$$

which yields for the distribution function

$$
\lim _{\Omega \rightarrow \infty} \bar{h}(y, \tau)=\frac{1}{\sqrt{\pi}} \Psi_{0}^{2}(y) .
$$

But this is exactly the linear noise approximation, which-in the new stochastic variable $y$-becomes time independent. We want to mention that Eq. (78) can be used as a starting point in order to transform the original master equation [Eq. (1a)] into a partial differential equation of infinite order, where the dependence on $\Omega$ is shown explicitly. This has been done in the work of van Kampen. ${ }^{8}$ In the limit $\Omega \rightarrow \infty$ one obtains a FokkerPlanck equation with time-dependent mean and variance, the solution of which is identical to Eq. (83). However, our solution Eq. (81) treats the full problem without any approximation and provides systematic corrections to the linear noise approximation. Corrections become especially necessary, if - due to the physical problem - the parameter $\Omega$ cannot be considered infinitely large. To give an example we refer to the Rayleigh piston problem. ${ }^{14}$

In concrete examples it is unlikely to find a closed expression replacing the infinite sum in Eq. (81a). Therefore one is led to replace the infinite sum by a finite sum. In doing this we can define the $k$ th approximation to the distribution function

$$
\bar{h}_{k}(y, \tau):=\sum_{n=0}^{k} b_{n} \Psi_{0}(y) \Psi_{n}(y) .
$$

Although it is in general difficult to answer questions concerning the positivity of the $k$ th approximation, one interesting conclusion can be drawn. From the orthogonality relations for the Hermite functions one easily finds that the $k$ th approximation $\bar{h}_{k}(y, \tau)$ gives the exact result for all moments $\left\langle y^{l}\right\rangle$ with $l=0,1, \ldots, k$. In particular one finds for the first moment

$$
\langle y\rangle=\int y \bar{h}(y, \tau) d y=\sqrt{\pi} b_{1}=\int y \bar{h}_{1}(y, \tau) d y,
$$

where we used

$$
\int y \Psi_{0}(y) \Psi_{n}(y) d y=\sqrt{\pi} \delta_{n, 1}
$$

One can easily verify that writing Eq. (85) in the original variable $x$ reproduces Eq. (68), which serves as a check for our general expression for the distribution function given by Eq. (81a).

\section{EXAMPLE}

For a simple check of our results presented in the previous sections let us consider the example of the Rayleigh piston. ${ }^{14}$ In this case the equilibrium distribution function $h^{\text {equ }}(x)$ is known to be a Gaussian distribution depending explicitly on the parameter $\Omega$

$$
h^{\mathrm{equ}}(x)=\left(\frac{\Omega-1}{\pi}\right)^{1 / 2} \exp \left[-x^{2}(\Omega-1)\right] .
$$

In what follows we want to show that our series expansion [Eq. (81a)] yields the correct equilibrium distribution function for all $\Omega$ whereas the linear noise approximation is valid only for $\Omega \rightarrow \infty$.

According to (81a) and (81b) we need the coefficients $c_{k, i}\left(x_{0}, \tau\right)$ for $\tau \rightarrow \infty$ in order to get the equilibrium distribution function. However, due to the rather involved structure of the recursion relation [Eq. (28)] it seems unlikely to find in this particular case a general expression for the time-dependent functions $\chi_{l, 2 l-k}$ from which the coefficients $c_{k, i}$ can then be derived [cf. Eq. (48)]. In order to avoid this difficulty we restrict ourselves to the following exercise: Rather than calculating directly the equilibrium distribution from the long time limit of the $\chi_{l, 2 l-k}$ 's and the corresponding $c_{k, i}$ 's we assume the equilibrium distribution $h^{\text {equ }}$, given by Eq. (87), to be valid. We then use this known $h^{\text {equ }}$ for calculating equilibrium averages via the relation

$$
\begin{aligned}
\lim _{\tau \rightarrow \infty}\left\langle f(x) \mid x_{0}\right\rangle_{\tau} & =\lim _{\tau \rightarrow \infty} \int f(x) h\left(x, \tau \mid x_{0}\right) d x \\
& =\int f(x) h^{\text {equ }}(x) d x .
\end{aligned}
$$


Expanding the rhs of Eq. (88) into a power series in $\Omega^{-1}$ we can compare it with the lhs of Eq. (88) or equivalently, with the long time limit of Eq. (15b). In this way we can determine the $\chi_{l, 2 l-k}\left(x_{0}, \tau\right)$ for $\tau \rightarrow \infty$ and, using Eq. (48), the coefficients $c_{k, i}\left(x_{0}, \tau\right)$ for $\tau \rightarrow \infty$ are obtained. As expected, it will turn out that these coeffi- cients determine a distribution function [see Eqs. (81a) and (81b)], which is the expansion of $h^{\text {equ }}(x)$ into Hermite functions.

Let us first calculate the equilibrium average as given on the rhs of Eq. (88). Expanding $f(x)$ into a Taylor series at $x=x_{0}=0$ and using Eq. (87) we obtain

$$
\int f(x) h^{\mathrm{equ}}(x) d x=f(0)+\sum_{l=1}^{\infty} \frac{1}{\Omega^{l}} \sum_{k=0}^{l-1} f^{(2 l-2 k)}(0)\left(\begin{array}{c}
l-1 \\
k
\end{array}\right) \frac{1}{(l-k) ! 4^{l-k}} .
$$

This expression can be compared with the long time limit of our nonequilibrium equation [Eq. (15b)] yielding

$\lim _{\tau \rightarrow \infty} \chi_{l, 2 l-2 k}\left(x_{0}, \tau\right)=: \chi_{l, 2 l-2 k}^{*}=\left(\begin{array}{c}l-1 \\ k\end{array}\right) \frac{\chi_{1,2}^{* l-k}}{(l-k) !}$

with

$$
\chi_{1,2}^{*}=\frac{1}{4}
$$

and

$$
\lim _{\tau \rightarrow \infty} \chi_{l, 2 l-2 k-l}\left(x_{0}, \tau\right)=0 \text { for } l \geq 1 .
$$

In order to obtain the coefficients $c_{k, i}\left(x_{0}, \tau\right)$ for $\tau \rightarrow \infty$ we have to cast Eqs. (90a) and (90b) into the following form [cf. Eq. (48)]:

$$
\chi_{l, 2 l-k}^{*}=\sum_{i=[k / 2]+1}^{2 k} c_{k, i}^{*} \frac{\chi_{1,2}^{* l-i}}{(l-i) !}
$$

with $l$-independent coefficients $c_{k, i}^{*}$. Comparing Eq. (91) with Eq. (90b) we find that $c_{k, i}^{*}=0$ for $k$ odd. Next, using the formula

$$
\sum_{i=0}^{k-1}\left(\begin{array}{c}
l-k \\
i+1
\end{array}\right]\left[\begin{array}{c}
k-1 \\
i
\end{array}\right)=\left(\begin{array}{c}
l-1 \\
k
\end{array}\right)
$$

one can easily verify that Eq. (90a) can be expressed by

$\chi_{l, 2 l-2 k}^{*}=\sum_{i=k+1}^{2 k}\left[\left[\begin{array}{c}k-1 \\ i-k-1\end{array}\right] \frac{\chi_{1,2}^{* i-k}}{(i-k) !}\right] \frac{\chi_{1,2}^{* l-i}}{(l-i) !}$.

If we now compare Eq. (93) with Eq. (91) we find

$c_{2 k, i}^{*}=\left(\begin{array}{c}k-1 \\ i-k-1\end{array}\right) \frac{1}{(i-k) ! 4^{i-k}}$, for $k+1 \leq i \leq 2 k$

and

$$
c_{2 k, i}^{*}=0, \text { for } i>2 k \text {. }
$$

We want to mention that only due to the requirement that $c_{k, i}^{*}$ has to be independent of $l$ one obtains a unique representation of $\chi_{l, 2 l-k}^{*}$ in the form of Eq. (91) and Eq. (93), respectively.

Finally, inserting Eqs. (94a) and (94b) into Eq. (81b) yields the coefficients $b_{n}\left(x_{0}, \tau\right)$ for $\tau \rightarrow \infty$

$$
\begin{aligned}
& \lim _{\tau \rightarrow \infty} b_{2 n}\left(x_{0}, \tau\right)=\frac{1}{\sqrt{\pi}} \frac{1}{n ! 4^{n}} \frac{1}{(\Omega-1)^{n}}, \\
& \lim _{\tau \rightarrow \infty} b_{2 n-1}\left(x_{0}, \tau\right)=0 .
\end{aligned}
$$

Combining Eqs. (95a) and (95b) and Eq. (81a) one can easily verify that for $\tau \rightarrow \infty$ the Gaussian distribution

$$
\lim _{\tau \rightarrow \infty} \bar{h}(y, \tau)=\left(\frac{\Omega-1}{\pi \Omega}\right]^{1 / 2} \exp \left[-y^{2} \frac{\Omega-1}{\Omega}\right)
$$

is obtained, which-restoring the original variable $x=y / \sqrt{\Omega}$ [see Eq. (78) for $\tau \rightarrow \infty$ ]-is identical to the equilibrium distribution function given by $\mathrm{Eq}$. (87).

\section{ACKNOWLEDGMENTS}

One of us (T.L.) wants to acknowledge a grant of the Max-Kade Foundation for his stay at the Massachusetts Institute of Technology.

\section{APPENDIX A}

In this Appendix we will show, that the derivatives of a composite function $f(g(x))$ are closely related to the cumulant expansion. A relation

$$
\exp \left[\sum_{l=1}^{\infty} \frac{\epsilon^{l}}{l !} q_{l}\right]=\sum_{l=0}^{\infty} \frac{\epsilon^{l}}{l !} p_{l}, \quad p_{0} \equiv 1
$$

is called the cumulant expansion of the power series on the rhs of Eq. (A1), and the coefficients $q_{l}$ are called cumulants. They can be uniquely expressed by the $p_{l}$ 's and vice versa. In particular for $p_{1}, p_{2}, p_{3}$, and $p_{4}$ we get

$$
\begin{aligned}
& p_{1}=q_{1}, \\
& p_{2}=q_{2}+q_{1}^{2}, \\
& p_{3}=q_{3}+3 q_{2} q_{1}+q_{1}^{3}, \\
& p_{4}=q_{4}+4 q_{3} q_{1}+3 q_{2}^{2}+6 q_{2} q_{1}^{2}+q_{1}^{4},
\end{aligned}
$$

and for more details we refer to Refs. 1 and 2 . If we replace in Eq. (A1) the $q_{l}$ 's by $q_{l} t$ we get

$$
\exp \left[\sum_{l=1}^{\infty} \frac{\epsilon^{l}}{l !} q_{l} t\right)=\sum_{l=0}^{\infty} \frac{\epsilon^{l}}{l !} p_{l}(t), p_{0}(t) \equiv 1,
$$

where $p_{l}(t)$ is a polynomial in $t$ of order $l$. So we can write

$$
p_{l}(t)=\sum_{k=1}^{l} p_{l, k} t^{k}, \quad l \geq 1
$$

with $t$-independent coefficients $p_{l, k}$. They are determined via Eqs. (A2a)-(A2d) replacing $q_{l}$ by $q_{l} t$ and comparing equal powers in $t$ with Eq. (A4). In general one can show that $p_{l, 1}=q_{l}$ and $p_{l, 1}=q_{1}^{l}$ for all $l \geq 1$. 
Let us next replace in Eq. (A3) the $q_{l}$ 's by the $l$ th derivative of an arbitrary function $g(x)$ and the variable $t$ by the differential operator $d / d y$. As a result we obtain two equivalent forms of a differential operator, and we want to consider its action on an arbitrary function $f(y)$. We obtain

$$
\begin{aligned}
\exp \left(\sum_{l=1}^{\infty} \frac{\epsilon^{l}}{l !} g^{(l)}(x)\right. & \left.\frac{d}{d y}\right] f(y) \\
& =f(y)+\sum_{l=1}^{\infty} \frac{\epsilon^{l}}{l !} \sum_{k=1}^{l} p_{l, k} f^{(k)}(y),
\end{aligned}
$$

where the superscript ( $l$ ) denotes the $l$ th derivative with respect to the argument. The lhs of Eq. (A5) can be simplified considerably, if we make use of the following relation:

$$
\exp \left[\alpha \frac{d}{d y}\right] f(y)=\sum_{l=0}^{\infty} \frac{\alpha^{l}}{l !} \frac{d^{l}}{d y^{l}} f(y)=f(y+\alpha) .
$$

If we select

$$
\alpha=\sum_{l=1}^{\infty} \frac{\epsilon^{l}}{l !} g^{(l)}(x)=g(x+\epsilon)-g(x)
$$

the lhs of Eq. (A5) becomes equivalent to $f(y+g(x$ $+\epsilon)-g(x))$. Next we consider Eq. (A5) for $y=g(x)$. The lhs of Eq. (A5) then becomes $f(g(x+\epsilon))$, which can be easily expanded in a Taylor series in $\epsilon$. In doing so we obtain

$$
\sum_{l=0}^{\infty} \frac{\epsilon^{l}}{l !} \frac{d^{l}}{d x^{l}} f(g(x))=f(g(x))+\sum_{l=1}^{\infty} \frac{\epsilon^{l}}{l !} \sum_{k=1}^{l} p_{l, k} f^{(k)}(g(x))
$$

or-comparing equal powers in $\epsilon-$

$$
\frac{d^{l}}{d x^{l}} f(g(x))=\sum_{k=0}^{l} p_{l, k} f^{(k)}(g(x)) \text {, with } p_{l, 0}=\delta_{l, 0} \text {. }
$$

Equation (A9) is the generalized chain rule for a composite function $f(g(x))$.

In Sec. III we considered the lth derivative of $f(\bar{x})$ with respect to $x_{0}$, where $\bar{x}$ was the solution of the macroscopic equation [Eq. (8)] and the dependence of $\bar{x}$ on the initial condition $x_{0}$ was given by Eq. (12). For this case we can express the $p_{l, k}$ 's by the independent variables $x_{0}$ and $\bar{x}$. In particular we obtain [see also (Eq. 19)]

$$
\begin{aligned}
& p_{1,1}=\bar{x}^{[1]}=\frac{\alpha_{1}(\bar{x})}{\alpha_{1}\left(x_{0}\right)}, \\
& p_{2,1}=\bar{x}^{[2]}=\frac{\alpha_{1}(\bar{x})}{\alpha_{1}^{2}\left(x_{0}\right)}\left[\alpha_{1}^{(1)}(\bar{x})-\alpha_{1}^{(1)}\left(x_{0}\right)\right], \\
& p_{2,2}=\left(\bar{x}^{[1]}\right)^{2}=\left[\frac{\alpha_{1}(\bar{x})}{\alpha_{1}\left(x_{0}\right)}\right]^{2}, \\
& p_{l, 1}=\bar{x}^{[l]}, p_{l, l}=\left(\bar{x}^{[1]}\right)^{l}=\left[\frac{\alpha_{1}(\bar{x})}{\alpha_{1}\left(x_{0}\right)}\right]^{l},
\end{aligned}
$$

(A10a) where a superscript in square brackets denotes a derivative with respect to $x_{0}$ and a superscript in parentheses denotes a derivative with respect to the argument.

\section{APPENDIX B}

Here we want to state two lemmata, which are used in Sec. IV and Sec. V, respectively. Lemma 1: Let $f_{0}$, $f_{1}, \ldots$ be a sequence of functions related by the following recursion relation:

$f_{l}(x)=-\int_{x}^{b} g^{\prime}(y) f_{l-1}(y) d y+\sum_{i=0}^{l} \int_{x}^{b} \frac{g^{l-i}(y)}{(l-i) !} h_{i}(y) d y$,

$f_{-1}(x) \equiv 0$

with arbitrary functions $g$ and $h_{0}, h_{1}, \ldots ; g^{\prime}=d g / d y$, then $f_{l}$ is explicitly given by

$$
f_{l}(x)=\sum_{i=0}^{l} \frac{g^{l-i}(x)}{(l-i) !} \int_{x}^{b} h_{i}(y) d y \text {. }
$$

This lemma can be easily proved by induction.

Lemma 2: Let $f$ be an arbitrary smooth function, which can be expanded in a Taylor series, then

$$
\begin{array}{r}
\int_{-\infty}^{\infty} f(x) \frac{e^{-\left[(x-y)^{2} / 4 \gamma\right]}}{\sqrt{4 \pi \gamma}} \frac{\boldsymbol{H} e_{n}((x-y) / \sqrt{4 \gamma})}{(\sqrt{4 \gamma})^{n}} d x \\
=\sum_{k=0}^{\infty} f^{(2 k+n)}(y) \frac{\gamma^{k}}{k !},
\end{array}
$$

where $\gamma$ is a positive constant and $H e_{n}$ denote the Hermite polynomials. Proof: Since $f$ can be expanded in a Taylor series we obtain

$$
\frac{\alpha}{\sqrt{\pi}} \int_{-\infty}^{\infty} f(x+y) e^{-(\alpha x)^{2}} d x=\sum_{k=0}^{\infty} \frac{f^{(2 k)}(y)}{k !} \frac{1}{\left(4 \alpha^{2}\right)^{k}}
$$

where $\alpha$ is a positive constant. Differentiating Eq. (B4) $n$ times with respect to $y$ yields

$$
\begin{aligned}
& \frac{\alpha}{\sqrt{\pi}} \int_{-\infty}^{\infty} \frac{d^{n}}{d y^{n}} f(x+y) e^{-(\alpha x)^{2}} d x \\
&=\sum_{k=0}^{\infty} \frac{f^{(2 k+n)}(y)}{k !} \frac{1}{\left(4 \alpha^{2}\right)^{k}}
\end{aligned}
$$

Since $d f(x+y) / d y=d f(x+y) / d x$ we get after integrating by part $n$ times

$$
\begin{aligned}
\frac{\alpha}{\sqrt{\pi}}(-1)^{n} \int_{-\infty}^{\infty} f(x+y) & \frac{d^{n}}{d x^{n}} e^{-(\alpha x)^{2}} d x \\
= & \sum_{k=0}^{\infty} \frac{f^{(2 k+n)}(y)}{k !} \frac{1}{\left(4 \alpha^{2}\right)^{k}} .
\end{aligned}
$$

Finally, since the Hermite polynomials are defined by ${ }^{13}$ 


$$
(-1)^{n} \frac{d^{n}}{d x^{n}} e^{-x^{2}}=e^{-x^{2}} H e_{n}(x)
$$

Eq. (B6) becomes

$$
\begin{aligned}
\int_{-\infty}^{\infty} f(x) \frac{\alpha}{\sqrt{\pi}} e^{-\alpha^{2}(x-y)^{2}} \alpha^{n} H e_{n}(\alpha(x-y)) d x \\
=\sum_{k=0}^{\infty} \frac{f^{(2 k+n)}(y)}{k !} \frac{1}{\left(4 \alpha^{2}\right)^{k}}
\end{aligned}
$$

which is identical to Eq. (B3), if we put $\alpha=1 / \sqrt{4 \gamma}$.

\section{APPENDIX C}

Although rearranging of a series is elementary, we want to suggest the following method for simplicity and select a particular example to demonstrate it. We want to rewrite Eq. (23) in the following form:

$$
\begin{array}{r}
\sum_{k=0}^{2 L-1} \sum_{m=0}^{s} \sum_{j=0}^{m}\left[\begin{array}{l}
s \\
m
\end{array}\right] f^{(2 L-k+j)}(\bar{x}) p_{m, j} \chi_{L, 2 L-k}^{[s-m]} \\
=\sum_{n=0}^{2 L} f^{(2 L+s-n)} c_{n}
\end{array}
$$

where the coefficients $c_{n}$ have to be determined. By calculating the highest and lowest derivative of $f$ on the lhs of Eq. (C1) one easily verifies the highest and lowest summation index on the rhs. Next we consider $\left(f^{(i)}\right)$ $i=0,1, \ldots$ as a set of orthonormal functions, which enables us to determine $c_{n}$

$$
c_{n}=\sum_{k=0}^{2 L-1} \sum_{m=0}^{s} \sum_{j=0}^{m}\left[\begin{array}{l}
s \\
m
\end{array}\right) \delta_{-k+j, s-n} p_{m, j} \chi_{L, 2 L-k}^{[s-m]} .
$$

In order to evaluate the Kronecker-delta we write Eq. (C2) as infinite sums by introducing the unit step function $\Theta$

$$
\begin{aligned}
c_{n} & =\sum_{k, m, j} \Theta(k) \Theta(2 L-1-k) \Theta(m)(s-m) \Theta(j) \Theta(m-j)\left[\begin{array}{c}
s \\
m
\end{array}\right] \delta_{j-s+n, k} p_{m, j} \chi_{L, 2 L-k}^{[s-m]} \\
& =\sum_{m, j} \Theta(j-s+n) \Theta(2 L-1-j+s-n) \Theta(m) \Theta(s-m) \Theta(j) \Theta(m-j)\left[\begin{array}{c}
s \\
m
\end{array}\right]_{p_{m, j}} \chi_{L, 2 L-j+s-n}^{[s-m]} .
\end{aligned}
$$

Due to Eq. (22) $\left(\chi_{l, 2 l-k} \equiv 0\right.$ for $\left.k<0\right)$ the first step function in Eq. (C3) is superfluous and the equation reduces to

$$
c_{n}=\sum_{j=0}^{2 L} \sum_{m=j}^{-1+s-n} \sum^{s}\left(\begin{array}{l}
s \\
m
\end{array}\right) p_{m, j} \chi_{L, 2 L-j+s-n}^{s-m]},
$$

where the asterisk indicates that the sum is assumed to be zero if the upper index is less than the lower index. We can simplify Eq. (C4) by considering the following cases.

(i) If $2 L-1+s-n \geq s$, the upper index of the first sum is $s$, since the second sum vanishes for $j>s$.

(ii) If $2 L-1+s-n \leq s-1$, we write in Eq. (C4)

$$
\sum_{j=0}^{2 L-s-n}=\sum_{j=0}^{s}-\sum_{j=2 L+s-n}^{s} .
$$

For $L \geq 1$ we see that the second sum in Eq. (C5) gives no contribution to (C4), since according to Eq. (22) we have the condition $\chi_{L, 2 L-k} \equiv 0$ for $k \geq 2 L$ and therefore Eq. (C4) can be written as

$$
c_{n}=\sum_{j=0}^{s} \sum_{m=j}^{s}\left[\begin{array}{l}
s \\
m
\end{array}\right] p_{m, j} \chi_{L, 2 L+s-j-n}^{[s-m]} .
$$

Inserting Eq. (C6) into (C4) finally yields

$$
\begin{aligned}
& \sum_{k=0}^{2 L-1} \sum_{m=0}^{s} \sum_{j=0}^{m}\left[\begin{array}{c}
s \\
m
\end{array}\right] f^{(2 L-k+j)}(\bar{x}) p_{m, j} \chi_{L, 2 L-k}^{[s-m]} \\
& =\sum_{k=0}^{2 L} f^{(2 L+s-k)} \sum_{j=0}^{s} \sum_{m=j}^{s}\left[\begin{array}{c}
s \\
m
\end{array}\right) p_{m, j} \chi_{L, 2 L+s-j-k}^{[s-m]} .
\end{aligned}
$$

It can be easily verified that according to the definition $\chi_{0,0} \equiv 1$ [see Eq. (22)] this equation is also valid for $L=0$.
*Present address: Department of Nuclear Engineering, Massachusetts Institute of Technology, Cambridge, MA 02139.

${ }^{1}$ I. Oppenheim, K. E. Shuler, and G. H. Weiss, Stochastic Processes in Chemical Physics: The Master Equation (MIT Cambridge, Mass., 1977).

${ }^{2}$ N. G. van Kampen, Stochastic Processes in Physics and Chemistry (North-Holland, Amsterdam, 1981).

${ }^{3}$ K. Andersen and K. E. Shuler, J. Chem. Phys. 40, 63 (1964).

${ }^{4}$ E. W. Montroll and K. E. Shuler, Adv. Chem. Phys. 1, 361 (1958).

${ }^{5}$ M. N. Barber and B. W. Ninham, Random and Restricted Walks (Gordon and Breach, New York, 1970).
6J. E. Moyal, J. R. Stat. Soc. B 11, 150 (1949).

${ }^{7}$ R. F. Pawula, Phys. Rev. 162, 186 (1967).

${ }^{8}$ N. G. van Kampen, Can. J. Phys. 39, 551 (1961).

${ }^{9}$ R. Kubo, K. Matsuo, and K. Kitahara, J. Stat. Phys. 9, 51 (1973).

${ }^{10}$ O. J. Eder and T. Lackner, Phys. Rev. A 28, 952 (1983).

${ }^{11}$ P. Hänggi and H. Thomas, Phys. Rep. 88, 207 (1982).

12O. J. Eder and T. Lackner, Phys. Rev. A 29, 799 (1984).

${ }^{13}$ I. N. Sneddon, Special Functions of Mathematical Physics and Chemistry (Oliver \& Boyd, Edinburgh and London, 1961).

${ }^{14}$ O. J. Eder, T. Lackner, and M. Posch, Phys. Rev. A 30, 1430 (1984). 